\title{
Inequalities for a generalized finite Hilbert transform of convex functions
}

\author{
Silvestru Sever Dragomir
}

\begin{abstract}
In this paper we obtain some new inequalities for a generalized finite Hilbert transform of convex functions. Applications for particular instances of finite Hilbert transforms are given as well.
\end{abstract}

\section{INTRODUCTION}

Finite Hilbert transform on the open interval $(a, b)$ is defined by

$$
(T f)(a, b ; t):=\frac{1}{\pi} P V \int_{a}^{b} \frac{f(\tau)}{\tau-t} \mathrm{~d} \tau:=\lim _{\varepsilon \rightarrow 0+}\left[\int_{a}^{t-\varepsilon}+\int_{t+\varepsilon}^{b}\right] \frac{f(\tau)}{\pi(\tau-t)} \mathrm{d} \tau,
$$

for $t \in(a, b)$ and for various classes of functions $f$ for which the above Cauchy Principal Value integral exists, see [14, Section 3.2] or [18, Lemma II.1.1].

Suppose that $I$ is an interval of real numbers with interior $\stackrel{\circ}{I}$ and $f: I \rightarrow \mathbb{R}$ is a convex function on $I$. Then $f$ is continuous on $\stackrel{\circ}{I}$ and has finite left and right derivatives at each point of $\stackrel{\circ}{I}$. Moreover, if $x, y \in \stackrel{\circ}{I}$ and $x<y$, then $f_{-}^{\prime}(x) \leq f_{+}^{\prime}(x) \leq f_{-}^{\prime}(y) \leq f_{+}^{\prime}(y)$ which shows that both $f_{-}^{\prime}$ and $f_{+}^{\prime}$ are nondecreasing function on $\stackrel{\circ}{I}$. It is also known that a convex function must be differentiable except for at most countably many points.

For a convex function $f: I \rightarrow \mathbb{R}$, the subdifferential of $f$ denoted by $\partial f$ is the set of all functions $\varphi: I \rightarrow[-\infty, \infty]$ such that $\varphi(\stackrel{\circ}{I}) \subset \mathbb{R}$ and

$$
f(x) \geq f(a)+(x-a) \varphi(a) \text { for any } x, a \in I .
$$

It is also well known that if $f$ is convex on $I$, then $\partial f$ is nonempty, $f_{-}^{\prime}$, $f_{+}^{\prime} \in \partial f$ and if $\varphi \in \partial f$, then

$$
f_{-}^{\prime}(x) \leq \varphi(x) \leq f_{+}^{\prime}(x) \text { for any } x \in \stackrel{\circ}{I} .
$$

2020 Mathematics Subject Classification. Primary: 26D15; Secondary: 26D10.

Key words and phrases. Finite Hilbert Transform, Convex functions, Integral inequalities.

Full paper. Received 30 November 2020, accepted 19 July 2021, available online 22 September 2021. 
In particular, $\varphi$ is a nondecreasing function. If $f$ is differentiable and convex on $\stackrel{\circ}{I}$, then $\partial f=\left\{f^{\prime}\right\}$.

The following result holds for the finite Hilbert transform of convex functions.

Theorem 1 (Dragomir et al., $2001[2])$. Let $f:(a, b) \rightarrow \mathbb{R}$ be a convex function on $(a, b)$. Then we have

$$
\begin{aligned}
& \frac{1}{\pi}\left[f(t) \ln \left(\frac{b-t}{t-a}\right)+f(t)-f(a)+f_{+}^{\prime}(t)(b-t)\right] \\
& \leq(T f)(a, b ; t) \\
& \leq \frac{1}{\pi}\left[f(t) \ln \left(\frac{b-t}{t-a}\right)+f(b)-f(t)+f_{-}^{\prime}(t)(t-a)\right],
\end{aligned}
$$

for all $t \in(a, b)$.

In particular, we have

$$
\begin{aligned}
& \frac{1}{\pi}\left[f\left(\frac{a+b}{2}\right)-f(a)+f_{+}^{\prime}\left(\frac{a+b}{2}\right)\left(\frac{b-a}{2}\right)\right] \\
& \leq(T f)\left(a, b ; \frac{a+b}{2}\right) \\
& \leq \frac{1}{\pi}\left[f(b)-f\left(\frac{a+b}{2}\right)+f_{-}^{\prime}\left(\frac{a+b}{2}\right)\left(\frac{b-a}{2}\right)\right] .
\end{aligned}
$$

For several recent papers devoted to inequalities for the finite Hilbert transform $(T f)$, see [3]-[13], [15]-[17] and [19,20].

We can naturally generalize the concept of Hilbert transform as follows.

For a continuous strictly increasing function $g:[a, b] \rightarrow[g(a), g(b)]$ that is differentiable on $(a, b)$ we define the following generalization of the finite Hilbert transform of a function $f:(a, b) \rightarrow \mathbb{C}$ by

$$
\begin{aligned}
\left(T_{g} f\right)(a, b ; t) & :=\frac{1}{\pi} P V \int_{a}^{b} \frac{f(\tau) g^{\prime}(\tau)}{g(\tau)-g(t)} \mathrm{d} \tau \\
& :=\lim _{\varepsilon \rightarrow 0+}\left[\int_{a}^{t-\varepsilon}+\int_{t+\varepsilon}^{b}\right] \frac{f(\tau) g^{\prime}(\tau)}{\pi[g(\tau)-g(t)]} \mathrm{d} \tau \\
& :=\frac{1}{\pi} \lim _{\varepsilon \rightarrow 0+}\left[\int_{a}^{t-\varepsilon} \frac{f(\tau) g^{\prime}(\tau)}{g(\tau)-g(t)} \mathrm{d} \tau+\int_{t+\varepsilon}^{b} \frac{f(\tau) g^{\prime}(\tau)}{g(\tau)-g(t)} \mathrm{d} \tau\right]
\end{aligned}
$$

for $t \in(a, b)$, provided the above $P V$ exists.

For $[a, b] \subset(0, \infty)$ and $g(t)=\ln t, t \in[a, b]$ we have the logarithmic finite Hilbert transform defined by

$$
\left(T_{\ln } f\right)(a, b ; t):=\frac{1}{\pi} \lim _{\varepsilon \rightarrow 0+}\left[\int_{a}^{t-\varepsilon} \frac{f(\tau)}{\tau \ln \left(\frac{\tau}{t}\right)} \mathrm{d} \tau+\int_{t+\varepsilon}^{b} \frac{f(\tau)}{\tau \ln \left(\frac{\tau}{t}\right)} \mathrm{d} \tau\right],
$$


where $t \in(a, b)$.

For $g(t)=\exp (\alpha t), t \in[a, b] \subset \mathbb{R}$ with $\alpha>0$ we have exponential finite Hilbert transform defined by

$$
\begin{aligned}
& \left(T_{\exp (\alpha)} f\right)(a, b ; t) \\
& :=\frac{1}{\pi} \lim _{\varepsilon \rightarrow 0+}\left[\int_{a}^{t-\varepsilon} \frac{f(\tau) \exp (\alpha \tau)}{\exp (\alpha \tau)-\exp (\alpha t)} \mathrm{d} \tau+\int_{t+\varepsilon}^{b} \frac{f(\tau) \exp (\alpha \tau)}{\exp (\alpha \tau)-\exp (\alpha t)} \mathrm{d} \tau\right]
\end{aligned}
$$

where $t \in(a, b)$.

For $[a, b] \subset(0, \infty)$ and $g(t)=t^{r}, t \in[a, b], r>0$, we have the positive $r$-power finite Hilbert transform defined by

(4) $\left(T_{r} f\right)(a, b ; t):=\frac{r}{\pi} \lim _{\varepsilon \rightarrow 0+}\left[\int_{a}^{t-\varepsilon} \frac{f(\tau) \tau^{r-1}}{\tau^{r}-t^{r}} \mathrm{~d} \tau+\int_{t+\varepsilon}^{b} \frac{f(\tau) \tau^{r-1}}{\tau^{r}-t^{r}} \mathrm{~d} \tau\right]$,

where $t \in(a, b)$.

Similarly, we can consider the function $g(t)=-t^{-p}, t \in[a, b] \subset(0, \infty)$, $p>0$, and then we have the negative p-power finite Hilbert transform

$$
\begin{aligned}
\left(T_{-p} f\right)(a, b ; t) & :=\frac{p}{\pi} \lim _{\varepsilon \rightarrow 0+}\left[\int_{a}^{t-\varepsilon} \frac{f(\tau) \tau^{-p-1}}{t^{-p}-\tau^{-p}} \mathrm{~d} \tau+\int_{t+\varepsilon}^{b} \frac{f(\tau) \tau^{-p-1}}{t^{-p}-\tau^{-p}} \mathrm{~d} \tau\right] \\
& =\frac{p t^{p}}{\pi} \lim _{\varepsilon \rightarrow 0+}\left[\int_{a}^{t-\varepsilon} \frac{f(\tau)}{\tau\left(\tau^{p}-t^{p}\right)} \mathrm{d} \tau+\int_{t+\varepsilon}^{b} \frac{f(\tau)}{\tau\left(\tau^{p}-t^{p}\right)} \mathrm{d} \tau\right],
\end{aligned}
$$

where $t \in(a, b)$.

For $[a, b] \subset\left[-\frac{\pi}{2 \rho}, \frac{\pi}{2 \rho}\right]$ and $g(t)=\sin (\rho t), t \in[a, b]$ where $\rho>0$, we have the $\rho$-sine finite Hilbert transform

(6) $\left(T_{\sin (\rho)} f\right)(a, b ; t)$

$$
:=\frac{\rho}{\pi} \lim _{\varepsilon \rightarrow 0+}\left[\int_{a}^{t-\varepsilon} \frac{f(\tau) \cos (\rho \tau)}{\sin (\rho \tau)-\sin (\rho t)} \mathrm{d} \tau+\int_{t+\varepsilon}^{b} \frac{f(\tau) \cos (\rho \tau)}{\sin (\rho \tau)-\sin (\rho t)} \mathrm{d} \tau\right],
$$

where $t \in(a, b)$.

For $g(t)=\sinh (\sigma t), t \in[a, b] \subset \mathbb{R}$ with $\sigma>0$ we have $\sigma$-sinh finite Hilbert transform

$$
\begin{aligned}
& \left(T_{\sinh (\sigma)} f\right)(a, b ; t) \\
& :=\frac{\sigma}{\pi} \lim _{\varepsilon \rightarrow 0+}\left[\int_{a}^{t-\varepsilon} \frac{f(\tau) \cosh (\sigma \tau)}{\sinh (\sigma \tau)-\sinh (\sigma t)} \mathrm{d} \tau+\int_{t+\varepsilon}^{b} \frac{f(\tau) \cosh (\sigma \tau)}{\sinh (\sigma \tau)-\sinh (\sigma t)} \mathrm{d} \tau\right],
\end{aligned}
$$

where $t \in(a, b)$. 
Similar transforms can be associated to the following functions as well:

$$
g(t)=\tan (\rho t), t \in[a, b] \subset\left[-\frac{\pi}{2 \rho}, \frac{\pi}{2 \rho}\right] \text { where } \rho>0,
$$

and

$$
g(t)=\tanh (\sigma t), t \in[a, b] \subset \mathbb{R} \text { with } \sigma>0 .
$$

Motivated by the above results, we establish in this paper some inequalities for the generalized finite Hilbert transform of convex functions on an interval. Applications for some particular instances of finite Hilbert transforms such as the ones from (2)-(7) are given as well.

\section{Main Results}

Consider the function $\mathbf{1}(t)=1, t \in(a, b)$. We need the following preliminary result.

Lemma 1. For a continuous strictly increasing function $g:[a, b] \rightarrow[g(a), g(b)]$ that is differentiable on $(a, b)$ we have

$$
\left(T_{g} \mathbf{1}\right)(a, b ; t)=\frac{1}{\pi} \ln \left(\frac{g(b)-g(t)}{g(t)-g(a)}\right), t \in(a, b) .
$$

We also have for $f:(a, b) \rightarrow \mathbb{C}$ that

$$
\left(T_{g} f\right)(a, b ; t)=\frac{1}{\pi} f(t) \ln \left(\frac{g(b)-g(t)}{g(t)-g(a)}\right)+\frac{1}{\pi} P V \int_{a}^{b} \frac{f(\tau)-f(t)}{g(\tau)-g(t)} g^{\prime}(\tau) \mathrm{d} \tau,
$$

for $t \in(a, b)$, provided that the $P V$ from the right hand side of the equality (9) exists.

Proof. We have

$$
\begin{aligned}
\left(T_{g} \mathbf{1}\right)(a, b ; t) & =\frac{1}{\pi} \lim _{\varepsilon \rightarrow 0+}\left[\int_{a}^{t-\varepsilon} \frac{g^{\prime}(\tau)}{g(\tau)-g(t)} \mathrm{d} \tau+\int_{t+\varepsilon}^{b} \frac{g^{\prime}(\tau)}{g(\tau)-g(t)} \mathrm{d} \tau\right] \\
& =\frac{1}{\pi} \lim _{\varepsilon \rightarrow 0+}\left[\ln \left|g(\tau)-g(t) \|_{a}^{t-\varepsilon}+\ln (g(\tau)-g(t))\right|_{t+\varepsilon}^{b}\right] \\
& =\frac{1}{\pi} \lim _{\varepsilon \rightarrow 0+}[\ln (g(t)-g(t-\varepsilon))-\ln (g(t)-g(a)) \\
& +\ln (g(b)-g(t))-\ln (g(t+\varepsilon)-g(t))] \\
& =\frac{1}{\pi} \ln \left(\frac{g(b)-g(t)}{g(t)-g(a)}\right)+\frac{1}{\pi} \lim _{\varepsilon \rightarrow 0+} \ln \left(\frac{g(t)-g(t-\varepsilon)}{g(t+\varepsilon)-g(t)}\right),
\end{aligned}
$$

for $t \in(a, b)$.

Since $g$ is differentiable, we have

$$
\lim _{\varepsilon \rightarrow 0+} \frac{g(t)-g(t-\varepsilon)}{g(t+\varepsilon)-g(t)}=\lim _{\varepsilon \rightarrow 0+} \frac{\frac{g(t)-g(t-\varepsilon)}{\varepsilon}}{\frac{g(t+\varepsilon)-g(t)}{\varepsilon}}=\frac{g^{\prime}(t)}{g^{\prime}(t)}=1,
$$


for $t \in(a, b)$, and by (10) we get $(8)$.

From the definition (1) we deduce

$$
\begin{aligned}
\left(T_{g} f\right)(a, b ; t) & :=\frac{1}{\pi} P V \int_{a}^{b} \frac{(f(\tau)-f(t)+f(t)) g^{\prime}(\tau)}{g(\tau)-g(t)} \mathrm{d} \tau \\
& =\frac{1}{\pi} P V \int_{a}^{b} \frac{(f(\tau)-f(t)) g^{\prime}(\tau) \mathrm{d} \tau}{g(\tau)-g(t)}+\frac{1}{\pi} P V \int_{a}^{b} \frac{f(t) g^{\prime}(\tau) \mathrm{d} \tau}{g(\tau)-g(t)} \\
& =\frac{1}{\pi} P V \int_{a}^{b} \frac{(f(\tau)-f(t)) g^{\prime}(\tau) \mathrm{d} \tau}{g(\tau)-g(t)}+\frac{1}{\pi} f(t) P V \int_{a}^{b} \frac{g^{\prime}(\tau) \mathrm{d} \tau}{g(\tau)-g(t)} \\
& =\frac{1}{\pi} f(t) \ln \left(\frac{g(b)-g(t)}{g(t)-g(a)}\right)+\frac{1}{\pi} P V \int_{a}^{b} \frac{(f(\tau)-f(t)) g^{\prime}(\tau) \mathrm{d} \tau}{g(\tau)-g(t)},
\end{aligned}
$$

for $t \in(a, b)$, which proves the identity (9).

If $g$ is a function which maps an interval $I$ of the real line to the real numbers, and is both continuous and injective then we can define the $g$ mean of two numbers $a, b \in I$ as

$$
M_{g}(a, b):=g^{-1}\left(\frac{g(a)+g(b)}{2}\right) .
$$

If $I=\mathbb{R}$ and $g(t)=t$ is the identity function, then $M_{g}(a, b)=A(a, b):=$ $\frac{a+b}{2}$, the arithmetic mean. If $I=(0, \infty)$ and $g(t)=\ln t$, then $M_{g}(a, b)=$ $G(a, b):=\sqrt{a b}$, the geometric mean. If $I=(0, \infty)$ and $g(t)=\frac{1}{t}$, then $M_{g}(a, b)=H(a, b):=\frac{2 a b}{a+b}$, the harmonic mean. If $I=(0, \infty)$ and $g(t)=$ $t^{p}, p \neq 0$, then $M_{g}(a, b)=M_{p}(a, b):=\left(\frac{a^{p}+b^{p}}{2}\right)^{1 / p}$, the power mean with exponent $p$. Finally, if $I=\mathbb{R}$ and $g(t)=\exp t$, then

$$
M_{g}(a, b)=L M E(a, b):=\ln \left(\frac{\exp a+\exp b}{2}\right),
$$

the LogMeanExp function.

Theorem 2. Assume that $g:[a, b] \rightarrow[g(a), g(b)]$ is a continuous strictly increasing function that is differentiable on $(a, b), f$ a function such that $f \circ g^{-1}:(g(a), g(b)) \rightarrow \mathbb{R}$ is a convex function on $(g(a), g(b))$. Then for $t \in(a, b)$ we have

$$
\begin{aligned}
\frac{1}{\pi}[f(t)- & \left.f(a)+[g(b)-g(t)] \frac{f_{+}^{\prime}(t)}{g^{\prime}(t)}\right] \\
\leq\left(T_{g} f\right)(a, b ; t) & -\frac{1}{\pi} f(t) \ln \left(\frac{g(b)-g(t)}{g(t)-g(a)}\right) \\
\leq & \frac{1}{\pi}\left[f(b)-f(t)+[g(t)-g(a)] \frac{f_{-}^{\prime}(t)}{g^{\prime}(t)}\right] .
\end{aligned}
$$

In particular, we have 


$$
\begin{aligned}
\frac{1}{\pi}\left[f\left(M_{g}(a, b)\right)-f(a)\right. & \left.+\frac{g(b)-g(a)}{2} \cdot \frac{f_{+}^{\prime}\left(M_{g}(a, b)\right)}{g^{\prime}\left(M_{g}(a, b)\right)}\right] \\
& \leq\left(T_{g} f\right)\left(a, b ; M_{g}(a, b)\right) \\
\leq \frac{1}{\pi}[f(b) & \left.-f\left(M_{g}(a, b)\right)+\frac{g(b)-g(a)}{2} \cdot \frac{f_{-}^{\prime}\left(M_{g}(a, b)\right)}{g^{\prime}\left(M_{g}(a, b)\right)}\right] .
\end{aligned}
$$

Proof. For $t, \tau \in(a, b)$ with $t \neq \tau$ we then have

$$
\frac{f(\tau)-f(t)}{g(\tau)-g(t)}=\frac{f \circ g^{-1}(g(\tau))-f \circ g^{-1}(g(t))}{g(\tau)-g(t)} .
$$

By the convexity of $f \circ g^{-1}$ we can state that for all $g(a) \leq c<d \leq g(b)$ we have

$$
\left(f \circ g^{-1}\right)_{-}^{\prime}(d) \geq \frac{\left(f \circ g^{-1}\right)(d)-\left(f \circ g^{-1}\right)(c)}{d-c} \geq\left(f \circ g^{-1}\right)_{+}^{\prime}(c) .
$$

Since $f \circ g^{-1}$ has lateral derivatives for $z \in(g(a), g(b))$ it follows $f$ has lateral derivatives in each point of $(a, b)$ and by the chain rule and the derivative of the inverse function,

$$
\left(f \circ g^{-1}\right)_{ \pm}^{\prime}(z)=\left(f_{ \pm}^{\prime} \circ g^{-1}\right)(z)\left(g^{-1}\right)^{\prime}(z)=\frac{\left(f_{ \pm}^{\prime} \circ g^{-1}\right)(z)}{\left(g^{\prime} \circ g^{-1}\right)(z)} .
$$

Let $t \in(a, b)$ and $t-a>\varepsilon>0$, then by (13)) and (14) we have

$$
\begin{aligned}
\frac{f \circ g^{-1}(g(\tau))-f \circ g^{-1}(g(t))}{g(\tau)-g(t)} & =\frac{f \circ g^{-1}(g(t))-f \circ g^{-1}(g(\tau))}{g(t)-g(\tau)} \\
& \geq \frac{\left(f_{+}^{\prime} \circ g^{-1}\right)(g(\tau))}{\left(g^{\prime} \circ g^{-1}\right)(g(\tau))}=\frac{f_{+}^{\prime}(\tau)}{g^{\prime}(\tau)},
\end{aligned}
$$

for $\tau \in(a, t-\varepsilon)$.

If we integrate the inequality (15) over $\tau$ on $(a, t-\varepsilon)$, we get by (12) that

$$
\begin{aligned}
\int_{a}^{t-\varepsilon} \frac{f(\tau)-f(t)}{g(\tau)-g(t)} g^{\prime}(\tau) \mathrm{d} \tau & \geq \int_{a}^{t-\varepsilon} \frac{f_{+}^{\prime}(\tau)}{g^{\prime}(\tau)} g^{\prime}(\tau) \mathrm{d} \tau \\
& =\int_{a}^{t-\varepsilon} f_{+}^{\prime}(\tau) \mathrm{d} \tau=f(t-\varepsilon)-f(a)
\end{aligned}
$$

for $t \in(a, b)$ and $t-a>\varepsilon>0$.

Let $t \in(a, b)$ and $b-t>\varepsilon>0$, then

$$
\frac{f \circ g^{-1}(g(\tau))-f \circ g^{-1}(g(t))}{g(\tau)-g(t)} \geq \frac{\left(f_{+}^{\prime} \circ g^{-1}\right)(g(t))}{\left(g^{\prime} \circ g^{-1}\right)(g(t))}=\frac{f_{+}^{\prime}(t)}{g^{\prime}(t)},
$$

for $\tau \in(t+\varepsilon, b)$.

This implies that

$$
\int_{t+\varepsilon}^{b} \frac{f(\tau)-f(t)}{g(\tau)-g(t)} g^{\prime}(\tau) \mathrm{d} \tau \geq \int_{t+\varepsilon}^{b} \frac{f_{+}^{\prime}(t)}{g^{\prime}(t)} g^{\prime}(\tau) \mathrm{d} \tau
$$




$$
=\frac{f_{+}^{\prime}(t)}{g^{\prime}(t)}[g(b)-g(t+\varepsilon)],
$$

for $t \in(a, b)$ and $b-t>\varepsilon>0$.

By adding the inequalities (16) and (17) we get

$$
\begin{aligned}
& \int_{a}^{t-\varepsilon} \frac{f(\tau)-f(t)}{g(\tau)-g(t)} g^{\prime}(\tau) \mathrm{d} \tau+\int_{t+\varepsilon}^{b} \frac{f(\tau)-f(t)}{g(\tau)-g(t)} g^{\prime}(\tau) \mathrm{d} \tau \\
& \geq f(t-\varepsilon)-f(a)+\frac{f_{+}^{\prime}(t)}{g^{\prime}(t)}[g(b)-g(t+\varepsilon)],
\end{aligned}
$$

for $t \in(a, b)$ and $\min \{b-t, t-a\}>\varepsilon>0$.

By taking the limit over $\varepsilon \rightarrow 0+$ in (18) we get

$$
P V \int_{a}^{b} \frac{f(\tau)-f(t)}{g(\tau)-g(t)} g^{\prime}(\tau) \mathrm{d} \tau \geq f(t)-f(a)+\frac{f_{+}^{\prime}(t)}{g^{\prime}(t)}[g(b)-g(t)],
$$

for $t \in(a, b)$.

By using the identity (9) we get the first inequality in (11).

Let $t \in(a, b)$ and $t-a>\varepsilon>0$, then by (13) and (14) we also have

$$
\begin{aligned}
\frac{f \circ g^{-1}(g(\tau))-f \circ g^{-1}(g(t))}{g(\tau)-g(t)} & =\frac{f \circ g^{-1}(g(t))-f \circ g^{-1}(g(\tau))}{g(t)-g(\tau)} \\
& \leq \frac{\left(f_{-}^{\prime} \circ g^{-1}\right)(g(t))}{\left(g^{\prime} \circ g^{-1}\right)(g(t))}=\frac{f_{-}^{\prime}(t)}{g^{\prime}(t)},
\end{aligned}
$$

for $\tau \in(a, t-\varepsilon)$.

If we integrate the inequality (20) over $\tau$ on $(a, t-\varepsilon)$, we get by (12) that

$$
\begin{aligned}
\int_{a}^{t-\varepsilon} \frac{f(\tau)-f(t)}{g(\tau)-g(t)} g^{\prime}(\tau) \mathrm{d} \tau & \leq \int_{a}^{t-\varepsilon} \frac{f_{-}^{\prime}(t)}{g^{\prime}(t)} g^{\prime}(\tau) \mathrm{d} \tau \\
& =\frac{f_{-}^{\prime}(t)}{g^{\prime}(t)}[g(t-\varepsilon)-g(a)],
\end{aligned}
$$

for $t \in(a, b)$ and $t-a>\varepsilon>0$.

Let $t \in(a, b)$ and $b-t>\varepsilon>0$, then

$$
\frac{f \circ g^{-1}(g(\tau))-f \circ g^{-1}(g(t))}{g(\tau)-g(t)} \leq \frac{\left(f_{-}^{\prime} \circ g^{-1}\right)(g(\tau))}{\left(g^{\prime} \circ g^{-1}\right)(g(\tau))}=\frac{f_{-}^{\prime}(\tau)}{g^{\prime}(\tau)},
$$

for $\tau \in(t+\varepsilon, b)$.

If we integrate the inequality (22) over $\tau$ on $(t+\varepsilon, b)$, we get

$$
\int_{t+\varepsilon}^{b} \frac{f(\tau)-f(t)}{g(\tau)-g(t)} g^{\prime}(\tau) \mathrm{d} \tau \leq \int_{t+\varepsilon}^{b} \frac{f_{-}^{\prime}(\tau)}{g^{\prime}(\tau)} g^{\prime}(\tau) \mathrm{d} \tau=f(b)-f(t+\varepsilon),
$$

for $t \in(a, b)$ and $b-t>\varepsilon>0$.

By adding the inequalities (21) and (23) we get

$$
\int_{a}^{t-\varepsilon} \frac{f(\tau)-f(t)}{g(\tau)-g(t)} g^{\prime}(\tau) \mathrm{d} \tau+\int_{t+\varepsilon}^{b} \frac{f(\tau)-f(t)}{g(\tau)-g(t)} g^{\prime}(\tau) \mathrm{d} \tau
$$




$$
\leq \frac{f_{-}^{\prime}(t)}{g^{\prime}(t)}[g(t-\varepsilon)-g(a)]+f(b)-f(t+\varepsilon),
$$

for $t \in(a, b)$ and $\min \{b-t, t-a\}>\varepsilon>0$.

By taking the limit over $\varepsilon \rightarrow 0+$ in (24) we get

$$
P V \int_{a}^{b} \frac{f(\tau)-f(t)}{g(\tau)-g(t)} g^{\prime}(\tau) \mathrm{d} \tau \leq f(b)-f(t)+\frac{f_{-}^{\prime}(t)}{g^{\prime}(t)}[g(t)-g(a)],
$$

for $t \in(a, b)$.

By using the identity (9) we obtain the second inequality in (11).

Remark 1. With the assumptions of Theorem 2, and if $f$ is differentiable on $(a, b)$, then we have

$$
\begin{aligned}
& \frac{1}{\pi}\left[f(t)-f(a)+[g(b)-g(t)] \frac{f^{\prime}(t)}{g^{\prime}(t)}\right] \\
& \leq\left(T_{g} f\right)(a, b ; t)-\frac{1}{\pi} f(t) \ln \left(\frac{g(b)-g(t)}{g(t)-g(a)}\right) \\
& \leq \frac{1}{\pi}\left[f(b)-f(t)+[g(t)-g(a)] \frac{f^{\prime}(t)}{g^{\prime}(t)}\right],
\end{aligned}
$$

for all $t \in(a, b)$.

In particular, we have

$$
\begin{aligned}
& \frac{1}{\pi}\left[f\left(M_{g}(a, b)\right)-f(a)+\frac{g(b)-g(a)}{2} \frac{f^{\prime}\left(M_{g}(a, b)\right)}{g^{\prime}\left(M_{g}(a, b)\right)}\right] \\
& \leq\left(T_{g} f\right)\left(a, b ; M_{g}(a, b)\right) \\
& \leq \frac{1}{\pi}\left[f(b)-f\left(M_{g}(a, b)\right)+\frac{g(b)-g(a)}{2} \frac{f^{\prime}\left(M_{g}(a, b)\right)}{g^{\prime}\left(M_{g}(a, b)\right)}\right] .
\end{aligned}
$$

We also have:

Theorem 3. Assume that $g:[a, b] \rightarrow[g(a), g(b)]$ is a continuous strictly increasing function that is differentiable on $(a, b)$ and $g_{+}^{\prime}(a)$ and $g_{-}(b)$ are finite. If $f \circ g^{-1}:(g(a), g(b)) \rightarrow \mathbb{R}$ is a convex function on $(a, b)$ and $f$ has finite lateral derivatives $f_{+}^{\prime}(a)$ and $f_{-}(b)$, then for $t \in(a, b)$ we have

$$
\begin{aligned}
\frac{f_{+}^{\prime}(a)}{\pi g_{+}^{\prime}(a)}[g(b)-g(a)] & \leq \frac{f(t)-f(a)}{\pi[g(t)-g(a)]}[g(b)-g(a)] \\
& \leq\left(T_{g} f\right)(a, b ; t)-\frac{1}{\pi} f(t) \ln \left(\frac{g(b)-g(t)}{g(t)-g(a)}\right) \\
& \leq \frac{f(b)-f(t)}{\pi[g(b)-g(t)]}[g(b)-g(a)] \\
& \leq \frac{f_{-}^{\prime}(b)}{\pi g_{-}^{\prime}(b)}[g(b)-g(a)] .
\end{aligned}
$$


In particular, for $t=M_{g}(a, b)$ we get

$$
\begin{aligned}
\frac{f_{+}^{\prime}(a)}{\pi g_{+}^{\prime}(a)}[g(b)-g(a)] & \leq \frac{2}{\pi}\left[f\left(M_{g}(a, b)\right)-f(a)\right] \\
& \leq\left(T_{g} f\right)\left(a, b ; M_{g}(a, b)\right) \\
& \leq \frac{2}{\pi}\left[f(b)-f\left(M_{g}(a, b)\right)\right] \leq \frac{f_{-}^{\prime}(b)}{\pi g_{-}^{\prime}(b)}[g(b)-g(a)] .
\end{aligned}
$$

Proof. We recall that if $\Phi: I \rightarrow \mathbb{R}$ is a continuous convex function on the interval of real numbers $I$ and $\alpha \in I$ then the divided difference function $\Phi_{\alpha}: I \backslash\{\alpha\} \rightarrow \mathbb{R}$

$$
\Phi_{\alpha}(t):=[\alpha, t ; \Phi]:=\frac{\Phi(t)-\Phi(\alpha)}{t-\alpha}
$$

is monotonic nondecreasing on $I \backslash\{\alpha\}$.

Using this property for the function $\Phi:(c, d) \rightarrow \mathbb{R}$, we have for $t \in(c, d)$ that

$$
\frac{\Phi(c)-\Phi(t)}{c-t} \leq \frac{\Phi(\tau)-\Phi(t)}{\tau-t} \leq \frac{\Phi(d)-\Phi(t)}{d-t}
$$

for any $\tau \in(c, d), \tau \neq t$.

By the gradient inequality for the convex function $\Phi$ we also have

$$
\frac{\Phi(t)-\Phi(c)}{t-c} \geq \Phi_{+}^{\prime}(c), \text { for } t \in(c, d)
$$

and

$$
\frac{\Phi(d)-\Phi(t)}{d-t} \leq \Phi_{-}(d), \text { for } t \in(c, d) .
$$

Therefore we have the following inequality

$$
\begin{aligned}
\Phi_{+}^{\prime}(c) & \leq \frac{\Phi(t)-\Phi(c)}{t-c} \leq \frac{\Phi(\tau)-\Phi(t)}{\tau-t} \\
& \leq \frac{\Phi(d)-\Phi(t)}{d-t} \leq \Phi_{-}(d),
\end{aligned}
$$

for $t, \tau \in(c, d)$ and $\tau \neq t$.

If we write the inequality (26) for the convex function $\Phi=f \circ g^{-1}$ and the interval $(g(a), g(b))$, we get

$$
\begin{aligned}
\frac{\left(f_{+}^{\prime} \circ g^{-1}\right)(g(a))}{\left(g_{+}^{\prime} \circ g^{-1}\right)(g(a))} & \leq \frac{\left(f \circ g^{-1}\right)(g(t))-\left(f \circ g^{-1}\right)(g(a))}{g(t)-g(a)} \\
& \leq \frac{\left(f \circ g^{-1}\right)(g(\tau))-\left(f \circ g^{-1}\right)(g(t))}{g(\tau)-g(t)} \\
& \leq \frac{\left(f \circ g^{-1}\right)(g(b))-\left(f \circ g^{-1}\right)(g(t))}{g(b)-g(t)} \\
& \leq \frac{\left(f_{-}^{\prime} \circ g^{-1}\right)(g(b))}{\left(g_{-}^{\prime} \circ g^{-1}\right)(g(b))},
\end{aligned}
$$


for $t, \tau \in(a, b)$ and $\tau \neq t$.

This is equivalent to

$$
\frac{f_{+}^{\prime}(a)}{g_{+}^{\prime}(a)} \leq \frac{f(t)-f(a)}{g(t)-g(a)} \leq \frac{f(\tau)-f(t)}{g(\tau)-g(t)} \leq \frac{f(b)-f(t)}{g(b)-g(t)} \leq \frac{f_{-}^{\prime}(b)}{g_{-}^{\prime}(b)},
$$

for $t, \tau \in(a, b)$ and $\tau \neq t$.

If we multiply with $g^{\prime}(\tau) \geq 0$ and take the $P V$ in (28), then we get

$$
\begin{aligned}
\frac{f_{+}^{\prime}(a)}{g_{+}^{\prime}(a)} \int_{a}^{b} g^{\prime}(\tau) \mathrm{d} \tau & \leq \frac{f(t)-f(a)}{g(t)-g(a)} \int_{a}^{b} g^{\prime}(\tau) \mathrm{d} \tau \\
& \leq P V \int_{a}^{b} \frac{f(\tau)-f(t)}{g(\tau)-g(t)} g^{\prime}(\tau) \mathrm{d} \tau \\
& \leq \int_{a}^{b} \frac{f(b)-f(t)}{g(b)-g(t)} g^{\prime}(\tau) \mathrm{d} \tau \leq \frac{f_{-}^{\prime}(b)}{g_{-}^{\prime}(b)} \int_{a}^{b} g^{\prime}(\tau) \mathrm{d} \tau,
\end{aligned}
$$

for $t \in(a, b)$, which is equivalent to

$$
\begin{aligned}
\frac{f_{+}^{\prime}(a)}{g_{+}^{\prime}(a)}[g(b)-g(a)] & \leq \frac{f(t)-f(a)}{g(t)-g(a)}[g(b)-g(a)] \\
& \leq P V \int_{a}^{b} \frac{f(\tau)-f(t)}{g(\tau)-g(t)} g^{\prime}(\tau) \mathrm{d} \tau \\
& \leq \frac{f(b)-f(t)}{g(b)-g(t)}[g(b)-g(a)] \leq \frac{f_{-}^{\prime}(b)}{g_{-}^{\prime}(b)}[g(b)-g(a)],
\end{aligned}
$$

for $t \in(a, b)$.

By the use of the identity (9) we obtain the desired result (25).

\section{Applications for $G A$-Convex Functions}

Let $I \subset(0, \infty)$ be an interval; a real-valued function $f: I \rightarrow \mathbb{R}$ is said to be GA-convex (concave) on $I$ if

$$
f\left(x^{1-\lambda} y^{\lambda}\right) \leq(\geq)(1-\lambda) f(x)+\lambda f(y)
$$

for all $x, y \in I$ and $\lambda \in[0,1]$. Since the condition (29) can be written as $f \circ \exp ((1-\lambda) \ln x+\lambda \ln y) \leq(\geq)(1-\lambda) f \circ \exp (\ln x)+\lambda f \circ \exp (\ln y)$, then we observe that $f: I \rightarrow \mathbb{R}$ is GA-convex (concave) on $I$ if and only if $f \circ \exp$ is convex (concave) on $\ln I:=\{\ln z, z \in I\}$. If $I=[a, b]$ then $\ln I=[\ln a, \ln b]$.

It is known that the function $f(x)=\ln (1+x)$ is $G A$-convex on $(0, \infty)$ [1].

For real and positive values of $x$, the Euler gamma function $\Gamma$ and its logarithmic derivative $\psi$, the so-called digamma function, are defined by

$$
\Gamma(x):=\int_{0}^{\infty} t^{x-1} e^{-t} \mathrm{~d} t \text { and } \psi(x):=\frac{\Gamma^{\prime}(x)}{\Gamma(x)} .
$$


It has been shown in [21] that the function $f:(0, \infty) \rightarrow \mathbb{R}$ defined by

$$
f(x)=\psi(x)+\frac{1}{2 x}
$$

is $G A$-concave on $(0, \infty)$ while the function $g:(0, \infty) \rightarrow \mathbb{R}$ defined by

$$
g(x)=\psi(x)+\frac{1}{2 x}+\frac{1}{12 x^{2}}
$$

is $G A$-convex on $(0, \infty)$.

If $[a, b] \subset(0, \infty)$ and the function $g:[\ln a, \ln b] \rightarrow \mathbb{R}$ is convex (concave) on $[\ln a, \ln b]$, then the function $f:[a, b] \rightarrow \mathbb{R}, f(t)=g(\ln t)$ is GA-convex (concave) on $[a, b]$.

Indeed, if $x, y \in[a, b]$ and $\lambda \in[0,1]$, then

$$
\begin{aligned}
f\left(x^{1-\lambda} y^{\lambda}\right) & =g\left(\ln \left(x^{1-\lambda} y^{\lambda}\right)\right)=g[(1-\lambda) \ln x+\lambda \ln y] \\
& \leq(\geq)(1-\lambda) g(\ln x)+\lambda g(\ln y)=(1-\lambda) f(x)+\lambda f(y),
\end{aligned}
$$

showing that $f$ is GA-convex (concave) on $[a, b]$.

Consider the following logarithmic finite Hilbert transform

$$
\left(T_{\ln } f\right)(a, b ; t):=\frac{1}{\pi} \lim _{\varepsilon \rightarrow 0+}\left[\int_{a}^{t-\varepsilon} \frac{f(\tau)}{\tau \ln \left(\frac{\tau}{t}\right)} \mathrm{d} \tau+\int_{t+\varepsilon}^{b} \frac{f(\tau)}{\tau \ln \left(\frac{\tau}{t}\right)} \mathrm{d} \tau\right],
$$

where $t \in(a, b) \subset(0, \infty)$.

Proposition 1. Assume that $f:[a, b] \subset(0, \infty) \rightarrow \mathbb{R}$ is GA-convex on $[a, b]$, then

$$
\begin{aligned}
& \frac{1}{\pi}\left[f(t)-f(a)+t f_{+}^{\prime}(t) \ln \left(\frac{b}{t}\right)\right] \\
& \leq\left(T_{\ln } f\right)(a, b ; t)-\frac{1}{\pi} f(t) \ln \left(\frac{\ln \left(\frac{b}{t}\right)}{\ln \left(\frac{t}{a}\right)}\right) \\
& \leq \frac{1}{\pi}\left[f(b)-f(t)+t f_{-}^{\prime}(t) \ln \left(\frac{t}{a}\right)\right],
\end{aligned}
$$

for all $t \in(a, b)$.

In particular,

$$
\begin{aligned}
& \frac{1}{\pi}\left[f(G(a, b))-f(a)+G(a, b) \ln \left(\sqrt{\frac{b}{a}}\right) f_{+}^{\prime}(G(a, b))\right] \\
& \leq\left(T_{g} f\right)(a, b ; G(a, b)) \\
& \leq \frac{1}{\pi}\left[f(b)-f(G(a, b))+G(a, b) \ln \left(\sqrt{\frac{b}{a}}\right) f_{-}^{\prime}(G(a, b))\right],
\end{aligned}
$$

where $G(a, b):=\sqrt{a b}$ is the geometric mean of $a, b>0$.

The proof follows by Theorem 2 for $g(t)=\ln t, t \in(a, b)$. 
Proposition 2. With the assumptions of Proposition 1 and if $f_{+}^{\prime}(a)$ and $f_{-}^{\prime}(b)$ are finite, then

$$
\begin{aligned}
\frac{a f_{+}^{\prime}(a)}{\pi} \ln \left(\frac{b}{a}\right) & \leq \frac{f(t)-f(a)}{\pi} \frac{\ln \left(\frac{b}{a}\right)}{\ln \left(\frac{t}{a}\right)} \\
& \leq\left(T_{\ln } f\right)(a, b ; t)-\frac{1}{\pi} f(t) \ln \left(\frac{\ln \left(\frac{b}{t}\right)}{\ln \left(\frac{t}{a}\right)}\right) \\
& \leq \frac{f(b)-f(t)}{\pi} \frac{\ln \left(\frac{b}{a}\right)}{\ln \left(\frac{b}{t}\right)} \leq \frac{b f_{-}^{\prime}(b)}{\pi} \ln \left(\frac{b}{a}\right),
\end{aligned}
$$

for any $t \in(a, b)$.

In particular,

$$
\begin{aligned}
\frac{a f_{+}^{\prime}(a)}{\pi} \ln \left(\frac{b}{a}\right) & \leq \frac{2}{\pi}[f(G(a, b))-f(a)] \\
& \leq\left(T_{\ln } f\right)(a, b ; G(a, b)) \\
& \leq \frac{2}{\pi}[f(b)-f(G(a, b))] \leq \frac{b f_{-}^{\prime}(b)}{\pi} \ln \left(\frac{b}{a}\right) .
\end{aligned}
$$

The proof follows by Theorem 3 for $g(t)=\ln t, t \in(a, b)$.

\section{Application for LogExp Convex Function}

We say that the function $f:[a, b] \rightarrow \mathbb{R}$ is a LogExp convex function on $[a, b]$ if $f \circ \ln$ is convex on the interval $[\exp a, \exp b]$, namely

$$
(f \circ \ln )((1-\lambda) u+\lambda v) \leq(1-\lambda)(f \circ \ln )(u)+\lambda(f \circ \ln )(v),
$$

for any $\lambda \in[0,1]$ and $u, v \in[\exp a, \exp b]$.

By taking $u=\exp t, v=\exp s, t, s \in[a, b]$, this is equivalent to

$$
f[\ln ((1-\lambda) \exp t+\lambda \exp s)] \leq(1-\lambda) f(t)+\lambda f(s),
$$

for any $\lambda \in[0,1]$ and $t, s \in[a, b]$.

For $g(t)=\exp (t), t \in[a, b] \subset \mathbb{R}$ we have the exponential finite Hilbert transform

$$
\begin{aligned}
& \left(T_{\exp } f\right)(a, b ; t) \\
& :=\frac{1}{\pi} \lim _{\varepsilon \rightarrow 0+}\left[\int_{a}^{t-\varepsilon} \frac{f(\tau) \exp (\tau)}{\exp (\tau)-\exp (t)} \mathrm{d} \tau+\int_{t+\varepsilon}^{b} \frac{f(\tau) \exp (\tau)}{\exp (\tau)-\exp (t)} \mathrm{d} \tau\right] \\
& =\frac{1}{\pi} \lim _{\varepsilon \rightarrow 0+}\left[\int_{a}^{t-\varepsilon} \frac{f(\tau)}{1-\exp (t-\tau)} \mathrm{d} \tau+\int_{t+\varepsilon}^{b} \frac{f(\tau)}{1-\exp (t-\tau)} \mathrm{d} \tau\right],
\end{aligned}
$$

where $t \in(a, b)$. 
Proposition 3. Assume that $f:[a, b] \rightarrow \mathbb{R}$ is LogExp convex function on $[a, b]$, then

$$
\begin{aligned}
& \frac{1}{\pi}\left[f(t)-f(a)+[\exp (b-t)-1] f_{+}^{\prime}(t)\right] \\
& \leq\left(T_{\exp } f\right)(a, b ; t)-\frac{1}{\pi} f(t) \ln \left(\frac{\exp (b-t)-1}{1-\exp (a-t)}\right) \\
& \leq \frac{1}{\pi}\left[f(b)-f(t)+[1-\exp (a-t)] f_{-}^{\prime}(t)\right],
\end{aligned}
$$

for any $t \in(a, b)$.

In particular,

$$
\begin{aligned}
& \frac{1}{\pi}\left[f(L M E(a, b))-f(a)+\frac{\exp (b)-\exp (a)}{\exp (b)+\exp (a)} f_{+}^{\prime}(L M E(a, b))\right] \\
& \leq\left(T_{g} f\right)(a, b ; L M E(a, b)) \\
& \leq \frac{1}{\pi}\left[f(b)-f(\operatorname{LME}(a, b))+\frac{\exp (b)-\exp (a)}{\exp (b)+\exp (a)} f_{-}^{\prime}(L M E(a, b))\right],
\end{aligned}
$$

where $\operatorname{LME}(a, b)=\ln \left(\frac{\exp a+\exp b}{2}\right)$ is the the LogMeanExp function of $a, b$.

The proof follows by Theorem 2 for $g(t)=\exp t, t \in(a, b)$.

Proposition 4. With the assumptions of Proposition 3 and if $f_{+}^{\prime}(a)$ and $f_{-}^{\prime}(b)$ are finite, then

$$
\begin{aligned}
\frac{f_{+}^{\prime}(a)}{\pi}[\exp (b-a)-1] & \leq \frac{f(t)-f(a)}{\pi}\left[\frac{\exp (b-a)-1}{\exp (t-a)-1}\right] \\
& \leq\left(T_{\exp } f\right)(a, b ; t)-\frac{1}{\pi} f(t) \ln \left(\frac{\exp (b-t)-1}{1-\exp (a-t)}\right) \\
& \leq \frac{f(b)-f(t)}{\pi}\left[\frac{1-g(a-b)}{1-g(t-b)}\right] \\
& \leq \frac{f_{-}^{\prime}(b)}{\pi}[1-\exp (a-b)],
\end{aligned}
$$

for $t \in(a, b)$.

In particular,

$$
\begin{aligned}
\frac{f_{+}^{\prime}(a)}{\pi}[\exp (b-a)-1] & \leq \frac{2}{\pi}[f(\operatorname{LME}(a, b))-f(a)] \\
& \leq\left(T_{\exp } f\right)(a, b ; \operatorname{LME}(a, b)) \\
& \leq \frac{2}{\pi}[f(b)-f(\operatorname{LME}(a, b))] \\
& \leq \frac{f_{-}^{\prime}(b)}{\pi}[1-\exp (a-b)] .
\end{aligned}
$$

The proof follows by Theorem 3 for $g(t)=\exp t, t \in(a, b)$. 
5. Application for Positive $p$-Convex Function

Let $p>0$. We say that the function $f:[a, b] \subset[0, \infty) \rightarrow \mathbb{R}$ is a positive $p$-convex function on $[a, b]$ if $f \circ(\cdot)^{1 / p}$ is convex on the interval $\left[a^{p}, b^{p}\right]$, namely

$$
f\left[((1-\lambda) u+\lambda v)^{1 / p}\right] \leq(1-\lambda) f\left(u^{1 / p}\right)+\lambda f\left(v^{1 / p}\right),
$$

for any $\lambda \in[0,1]$ and $u, v \in\left[a^{p}, b^{p}\right]$.

By taking $u=t^{p}, v=s^{p}, t, s \in[a, b]$, this is equivalent to, see also [22]

$$
f\left[\left((1-\lambda) t^{p}+\lambda s^{p}\right)^{1 / p}\right] \leq(1-\lambda) f(t)+\lambda f(s),
$$

for any $\lambda \in[0,1]$ and $t, s \in[a, b]$.

For $[a, b] \subset(0, \infty)$ and $g(t)=t^{p}, t \in[a, b], p>0$, we consider the positive p-power Hilbert transform

$$
\left(T_{p} f\right)(a, b ; t):=\frac{p}{\pi} \lim _{\varepsilon \rightarrow 0+}\left[\int_{a}^{t-\varepsilon} \frac{f(\tau) \tau^{p-1}}{\tau^{p}-t^{p}} \mathrm{~d} \tau+\int_{t+\varepsilon}^{b} \frac{f(\tau) \tau^{p-1}}{\tau^{p}-t^{p}} \mathrm{~d} \tau\right],
$$

where $t \in(a, b)$.

Proposition 5. Assume that $f:[a, b] \rightarrow \mathbb{R}$ is positive p-convex function on $[a, b]$, then

$$
\begin{aligned}
& \frac{1}{\pi}\left[f(t)-f(a)+\frac{b^{p}-t^{p}}{p t^{p-1}} f_{+}^{\prime}(t)\right] \\
& \leq\left(T_{p} f\right)(a, b ; t)-\frac{1}{\pi} f(t) \ln \left(\frac{b^{p}-t^{p}}{t^{p}-a^{p}}\right) \\
& \leq \frac{1}{\pi}\left[f(b)-f(t)+\frac{t^{p}-a^{p}}{p t^{p-1}} f_{-}^{\prime}(t)\right],
\end{aligned}
$$

for $t \in(a, b)$.

In particular, we have

$$
\begin{aligned}
& \frac{1}{\pi}\left[f\left(M_{p}(a, b)\right)-f(a)+\frac{b^{p}-a^{p}}{2 p M_{p}^{p-1}(a, b)} f_{+}^{\prime}\left(M_{p}(a, b)\right)\right] \\
& \leq\left(T_{g} f\right)\left(a, b ; M_{p}(a, b)\right) \\
& \leq \frac{1}{\pi}\left[f(b)-f\left(M_{p}(a, b)\right)+\frac{b^{p}-a^{p}}{2 p M_{p}^{p-1}(a, b)} f_{-}^{\prime}\left(M_{p}(a, b)\right)\right],
\end{aligned}
$$

where $M_{p}(a, b):=\left(\frac{a^{p}+b^{p}}{2}\right)^{1 / p}$.

The proof follows by Theorem 2 for $g(t)=t^{p}, t \in[a, b]$. 
Proposition 6. With the assumptions of Proposition 5 and if $f_{+}^{\prime}(a)$ and $f_{-}^{\prime}(b)$ are finite, then

$$
\begin{aligned}
\frac{b^{p}-a^{p}}{p \pi a^{p-1}} f_{+}^{\prime}(a) & \leq \frac{f(t)-f(a)}{\pi}\left(\frac{b^{p}-a^{p}}{t^{p}-a^{p}}\right) \\
& \leq\left(T_{p} f\right)(a, b ; t)-\frac{1}{\pi} f(t) \ln \left(\frac{b^{p}-t^{p}}{t^{p}-a^{p}}\right) \\
& \leq \frac{f(b)-f(t)}{\pi}\left(\frac{b^{p}-a^{p}}{b^{p}-t^{p}}\right) \leq \frac{b^{p}-a^{p}}{p \pi a^{p-1}} f_{-}^{\prime}(b) .
\end{aligned}
$$

In particular,

$$
\begin{aligned}
\frac{b^{p}-a^{p}}{p \pi a^{p-1}} f_{+}^{\prime}(a) & \leq \frac{2}{\pi}\left[f\left(M_{p}(a, b)\right)-f(a)\right] \\
& \leq\left(T_{p} f\right)\left(a, b ; M_{p}(a, b)\right) \\
& \leq \frac{2}{\pi}\left[f(b)-f\left(M_{p}(a, b)\right)\right] \leq \frac{b^{p}-a^{p}}{p \pi a^{p-1}} f_{-}^{\prime}(b) .
\end{aligned}
$$

The proof follows by Theorem 3 for $g(t)=t^{p}, t \in(a, b)$.

Similar results may be stated for negative $p$-power convex functions, namely for $g(t)=-\frac{1}{t^{p}}, t \in[a, b] \subset(0, \infty)$. The details are omitted.

\section{REFERENCES}

[1] G. D. Anderson, M. K. Vamanamurthy, M. Vuorinen, Generalized convexity and inequalities, Journal of Mathematical Analysis and Applications, 335 (2007), 1294-1308.

[2] N. M. Dragomir, S. S. Dragomir, P. M. Farrell, Some inequalities for the finite Hilbert transform in Inequality Theory and Applications, vol. I, 113-122, Nova Science Publishers, Huntington, NY, 2001.

[3] N. M. Dragomir, S. S. Dragomir, P. M. Farrell, Approximating the finite Hilbert transform via trapezoid type inequalities, Computational Mathematics and Applications, 43 (10-11) (2002), 1359-1369.

[4] N. M. Dragomir, S. S. Dragomir, P. M. Farrell, G. W. Baxter, On some new estimates of the finite Hilbert transform, Libertas Mathematica, 22 (2002), 65-75.

[5] N. M. Dragomir, S. S. Dragomir, P. M. Farrell, G. W. Baxter, A quadrature rule for the finite Hilbert transform via trapezoid type inequalities, Journal of Applied Mathematics and Computers, 13 (1-2) (2003), 67-84.

[6] N. M. Dragomir, S. S. Dragomir, P. M. Farrell, G. W. Baxter, A quadrature rule for the finite Hilbert transform via midpoint type inequalities in Fixed Point Theory and Applications, vol. 5, 11-22, Nova Science Publishers, Hauppauge, NY, 2004.

[7] S. S. Dragomir, Inequalities for the Hilbert transform of functions whose derivatives are convex, Journal of Korean Mathematical Society, 39 (5) (2002), 709-729.

[8] S. S. Dragomir, Approximating the finite Hilbert transform via an Ostrowski type inequality for functions of bounded variation, Journal of Inequalities in Pure and Applied Mathematics, 3 (4) (2002), Article 51, 19 pages.

[9] S. S. Dragomir, Approximating the finite Hilbert transform via Ostrowski type inequalities for absolutely continuous functions, Bulletin of Korean Mathematical Society, 39 (4) (2002), 543-559. 
[10] S. S. Dragomir, Some inequalities for the finite Hilbert transform of a product, Communications of Korean Mathematical Society, 18 (1) (2003), 39-57.

[11] S. S. Dragomir, Sharp error bounds of a quadrature rule with one multiple node for the finite Hilbert transform in some classes of continuous differentiable functions, Taiwanese Journal of Mathematics, 9 (1) (2005), 95-109.

[12] S. S. Dragomir, The perturbed median principle for integral inequalities with applications in Nonlinear Analysis and Variational Problems, Springer Optimization and Its Applications, Springer, New York, 35 (2010), 53-63.

[13] S. S. Dragomir, Inequalities and approximations for the Finite Hilbert transform: a survey of recent results, Preprint, RGMIA Research Report Collection 21 (2018), Art. 30, 90 pp. [Online http://rgmia.org/papers/v21/v21a30.pdf] .

[14] F. D. Gakhov, Boundary Value Problems (English translation), Pergamon Press, Oxford, 1966.

[15] W. Liu, X. Gao, Approximating the finite Hilbert transform via a companion of Ostrowski's inequality for function of bounded variation and applications, Applied Mathematics and Computation, 247 (2014), 373-385.

[16] W. Liu, X. Gao, Y. Wen, Approximating the finite Hilberttransform via some companions of Ostrowski's inequalities, Bulletin Malaysian Mathematical Sciences Society, 39 (4) (2016), 1499-1513.

[17] W. Liu, N. Lu, Approximating the finite Hilbert transform via Simpson type inequalities and applications, Politehnica University Bucharest Science Bulletin Series. A Applied Mathematics and Physics, 77 (3) (2015), 107-122.

[18] S. G. Mikhlin, S. Prössdorf, Singular Integral Operators (English translation), Springer Verlag, Berlin, 1986.

[19] S. Wang, X. Gao, N. Lu, A quadrature formula in approximating the finite Hilbert transform via perturbed trapezoid type inequalities, Journal Computational Analysis and Applications, 22 (2) (2017), 239-246.

[20] S. Wang, N. Lu, X. Gao, A quadrature rule for the finite Hilbert transform via Simpson type inequalities and applications, Journal Computational Analysis and Applications, 22 (2) (2017), 229-238.

[21] X.-M. Zhang, Y.-M. Chu, X.-H. Zhang, The Hermite-Hadamard type inequality of GA-convex functions and its application, Journal of Inequalities and Applications, 2010, Article ID: 507560, 11 pages.

[22] K. S. Zhang, J. P. Wan, p-convex functions and their properties, Pure and Applied Mathematics, 23 (1) (2007), 130-133.

\section{Silvestru Sever Dragomir}

Mathematics, College of Engineering \& Science

Victoria University, PO Box 14428

Melbourne City, MC 8001

Australia

Secondary address:

DST-NRF CENTRE of Excellence in the

Mathematical and Statistical Sciences

School of Computer Science \& Applied Mathematics

UNIVERSity OF THE WitWATERSRAND

Private Bag 3, Johannesburg 2050

South Africa

E-mail address: sever.dragomir@vu.edu.au 